\title{
Are we on the same page? Exploring the role of the geriatrician in the care of the older surgical patient from the perspective of surgeons and geriatricians
}

\author{
Authors: Sarah Howie ${ }^{A}$ and Anthea Tinker ${ }^{B}$
}

There is increasing recognition that the ageing population represents a challenge to existing surgical services. National reports recommend that geriatricians proactively review older surgical patients to improve care and outcomes. However, this approach has not been widely translated into practice. A qualitative study was conducted using 12 semi-structured interviews of surgeons and geriatricians to explore the role of the geriatrician in the care of older surgical patients. Participants agreed that the current system did not meet the needs of older surgical patients. Geriatricians valued their holistic way of working but these generalist skills can overlap with other specialties, seen by some as wasting resources. Three models of care were proposed, with the ownership and location of the patient as well as the role of education being the key variables. The main obstacle preventing integrated working was the concern of de-skilling the surgeons, narrowing their role to that of a 'technician'. Other barriers included loss of autonomy; lack of evidence; and a lack of recognition of the need for a geriatrician. There is acceptance that closer working practices are necessary to meet the needs of this complex patient group but a lack of evidence, together with significant human factors, are challenges that must be addressed to realise this aim.

KEYWORDS: Surgical liaison, orthogeriatrics, high-risk surgery, older patient, collaborative care

\section{Introduction}

Increasingly, the ageing population and its associated medical complexity represent a challenge to surgical services. Reports suggest that outcomes for high-risk older surgical patients could be improved upon, ${ }^{1-4}$ with the recommendation of the routine involvement of geriatricians. Despite the widely recognised improvements brought about by involving geriatricians in the care of patients with hip fractures, ${ }^{5-7}$ geriatrician input in other areas of surgery has been not been widely implemented. ${ }^{8,9}$ It has been suggested that a lack of recognition of the value of the

Authors: ${ }^{\text {A }}$ consultant orthogeriatrician, Kingston Hospital NHS Foundation Trust, London, UK; ${ }^{\text {B }}$ professor of social gerontology, King's College London, London, UK comprehensive geriatric assessment outside of geriatrics and limited evidence to support its use in surgical pathways could be contributing factors, ${ }^{8}$ but these hypotheses have not been tested.

To better understand why these recommendations are not being implemented, here we highlight different perspectives held by geriatricians and surgeons regarding the role of the geriatrician in the care of older surgical patients. We also identify some of the potential barriers that could be preventing effective collaboration between the two specialties.

\section{Aims and objectives}

The aim of this study was to explore the role of the geriatrician in the care of older surgical patients from the perspective of surgeons and geriatricians. The four key objectives were to:

> Evaluate the current system of providing geriatric input into the care of older surgical patients.

> Examine which particular skills the geriatrician can add.

$>$ Ascertain how the current configuration of care could be improved.

> Identify potential barriers preventing surgeons and geriatricians from working together in a more integrated way.

\section{Methods}

This qualitative study collected data using semistructured interviews with six geriatricians and six surgeons in the south-west Thames region. The geriatricians were all consultants, two of whom were orthogeriatricians, working at five different trusts. Five of the surgeons were consultants and the sixth was a ST6-level surgical trainee. The surgeons specialised in vascular, urology, upper gastrointestinal, colorectal, general and orthopaedic surgery, and worked at three different trusts.

The geriatricians were recruited via a generic email sent to the regional British Geriatrics Society. The first four of 16 positive replies were interviewed. In addition, to explore some anticipated themes, a geriatrician with many years of experience was chosen along with a geriatrician involved in setting up a surgical liaison service. The surgeons were recruited via two geriatricians who acted as gatekeepers. Of the eight who agreed to participate, six were chosen to ensure the main subspecialties within surgery were covered and that more than one trust was represented.

Eleven participants were interviewed face-to-face, whereas one interview was conducted via telephone. An information 


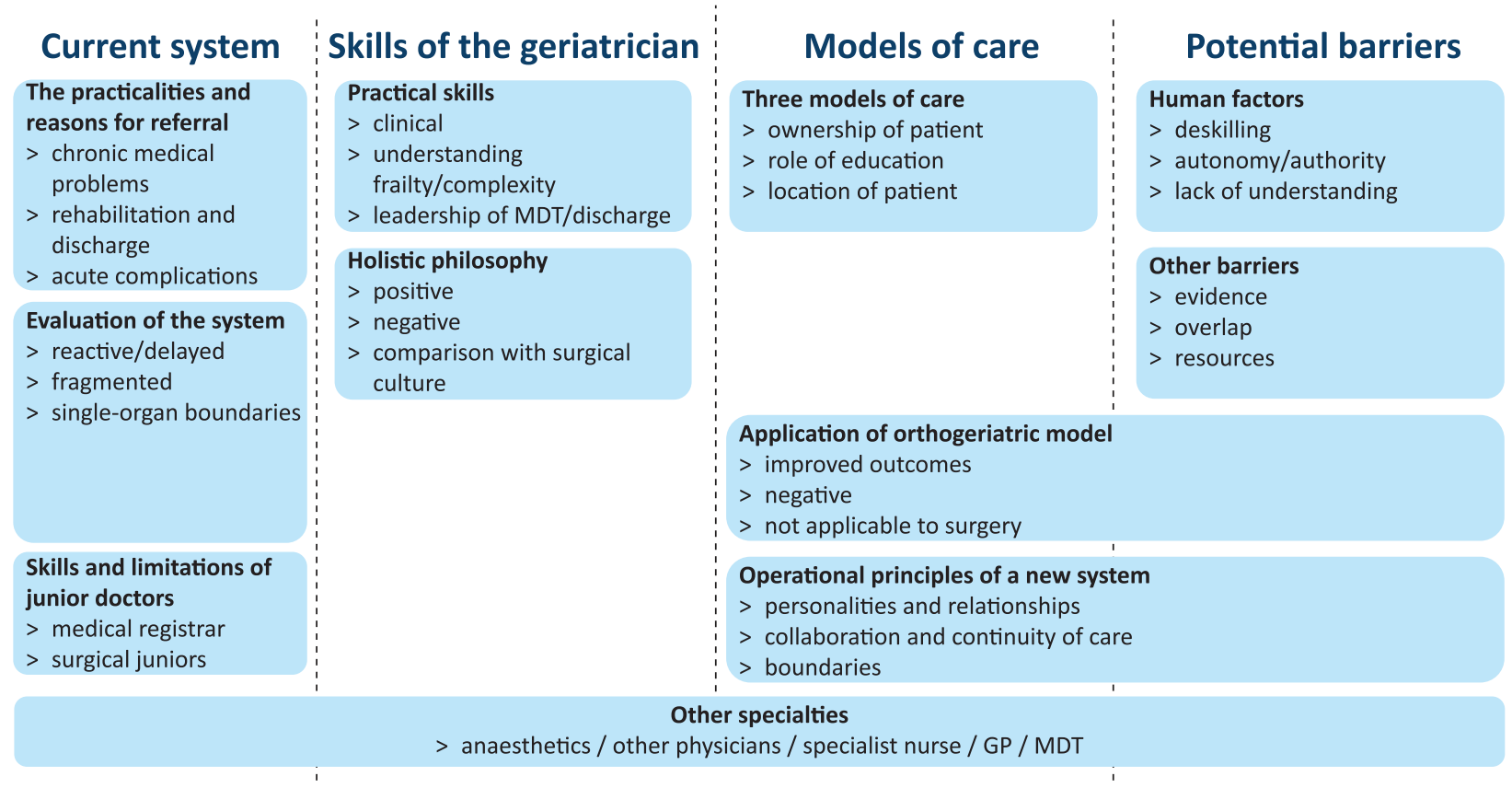

Fig 1. Coding results organised into key themes and categories mapped to the four objectives. $A=$ anaesthetist; $G=$ geriatrician; $G P=$ general practitioner; $\mathrm{MDT}=$ multidisciplinary team; $\mathrm{OP}=$ other physicians; $\mathrm{S}=$ surgeon

sheet was given and written consent was obtained. Low-risk ethics approval was given by the King's College London ethics committee on 12 November 2014. The interviews were directed using a topic guide covering the four objectives but with as many open questions as possible to encourage the clinicians to give their own opinion with as little influence from the researcher as possible. ${ }^{10}$ The interviews lasted between 29 and 55 min and occurred between January and May 2015.

All interviews were audio-recorded and transcribed verbatim. A grounded theory approach was used to analyse the data and the computer software NVivo Student 10 was used to assist the first stages of data analysis. Data coding was carried out after each interview to enable gaps in the data to be filled with subsequent interviews. Initial coding was conducted line by line, assigning short, simple terms to reflect what was being said. By constant comparison of the data, these were refined to ensure consistency throughout. This was followed by the introduction of more conceptual codes, which were categorised to explore the relationships between them. Finally, broader themes were identified in relation to the four key objectives.
This method of data collection enabled the views of clinicians to be explored in an amount of depth that revealed themes that other methods would not otherwise have done. By collecting and analysing the data simultaneously, new areas of enquiry could be explored as they presented themselves, allowing concepts and theories to emerge from the data as one integrated process.

\section{Results}

The themes and categories, which were mapped to the four objectives, are illustrated in Fig 1. The first objective revealed two themes: the practicalities of the referral process and the evaluation of the current system. The second identified the practical skills of the geriatrician as well as the philosophy of their working practice. The third objective elicited three different amendments to the current model of care based on the ownership and location of the patient, along with the role of education, illustrated in Figs 2-5. Finally, the potential barriers to better collaboration were divided into human factors and other barriers. The key points within these themes are described below.

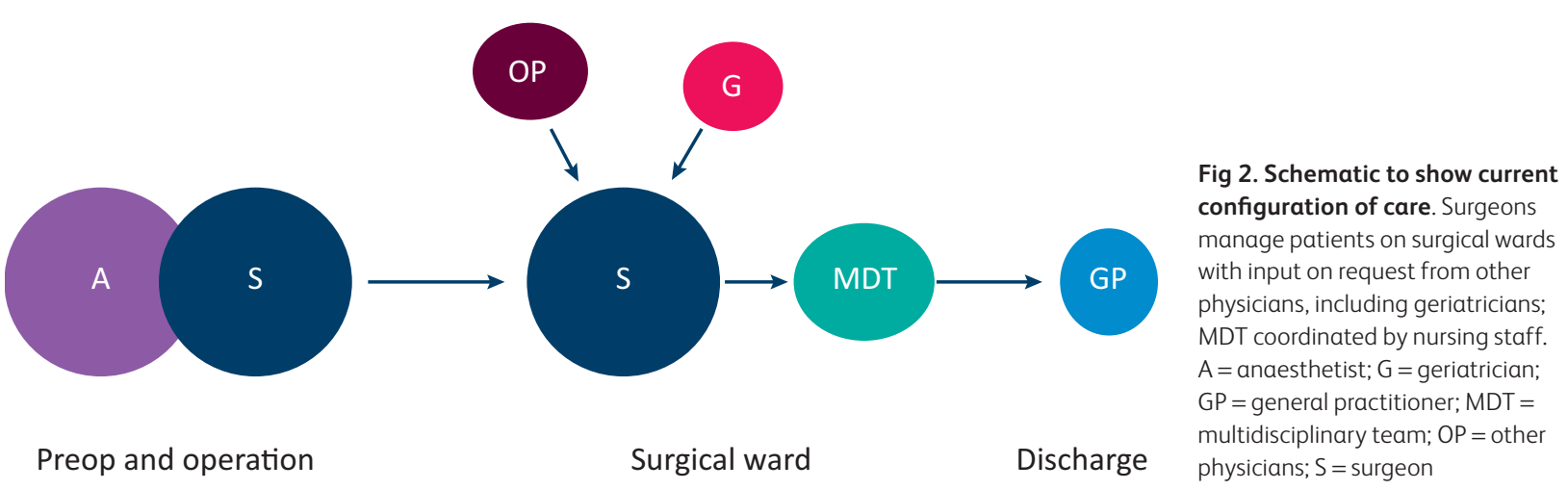


Fig 3. First amended model of care. An expanded role for surgeons, trained to manage medical problems in complex older patients; geriatricians have an advisory and teaching role. $A=$ anaesthetist; $G=$ geriatrician; $\mathrm{GP}=$ general practitioner; $\mathrm{MDT}=$ multidisciplinary team; $\mathrm{OP}=$ other physicians; $\mathrm{S}=$ surgeon

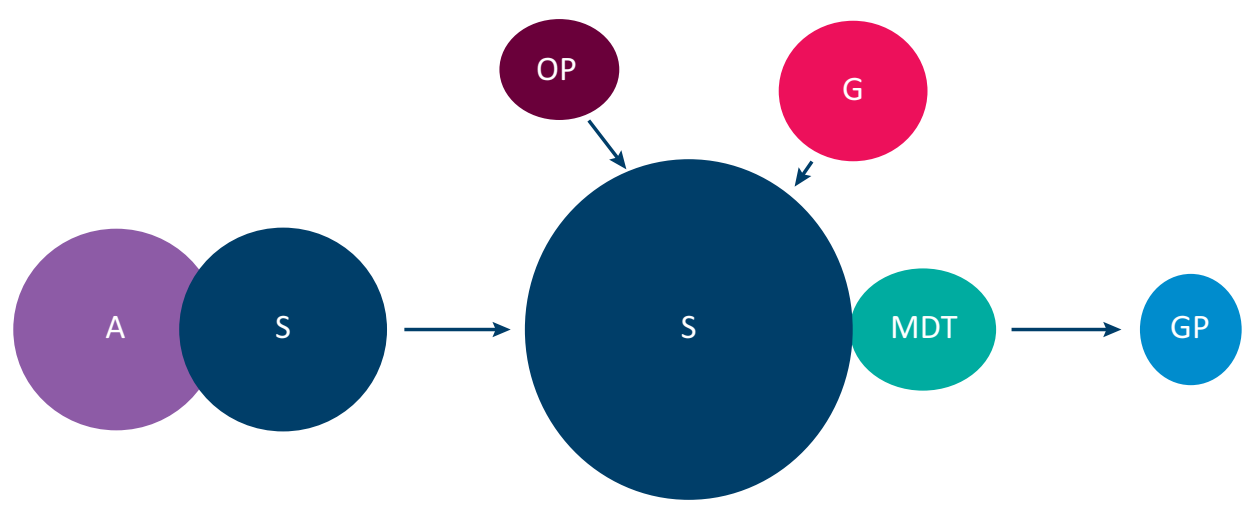

Preop and operation

\section{The current system}

The configuration of the current system is illustrated in Fig 2. All trusts required written referral to obtain a geriatrician's opinion. The reasons for referral fell into three categories: chronic medical problems; rehabilitation and discharge; and acute complications.

In evaluating the current system, many criticisms referred to reactive and delayed care. Whereas some geriatricians referred to the patients being 'in extremis' (Interview 10) and the detrimental impact on patients of delayed input, only the surgical trainee hinted that the patient's physical condition might deteriorate. The surgeons either focused more on the implications for length of stay or did not reflect specifically on the impact on patient care. Several references were made to the fact that multiple specialties are often involved at different stages of the patient's journey, often leading to fragmented care.

Eleven participants felt that the current system did not meet the needs of older surgical patients, with most stating clearly that it was not sufficient 'by any stretch of the imagination' (Interview 4 , vascular surgeon).

\section{The skills of the geriatrician}

The second objective sought to identify the particular skills of geriatricians relevant to older surgical patients. Many participants listed the areas one might expect: dealing with multiple comorbidities, polypharmacy, end-of-life care, understanding frailty, and leading the multidisciplinary team (MDT). However, some surgeons felt that there was no particular difference between geriatricians and any other physician. Many participants emphasised the benefit of the geriatrician's generalist expertise in managing multimorbidities; however, some expressed reservations regarding their perceived lack of specialist skills.

All the geriatricians identified positively with a holistic philosophy of working, but not all surgeons recognised it. The potential for the generalist to overlap with other specialties was felt by some to be wasteful of limited resources. By direct contrast, the task-driven 'surgical culture' (Interview 10) was universally reported in a negative light and was felt to have worsened over recent times. One surgeon suggested that they did not have time for compassion in the way that geriatricians did. Thus, there is clearly a difference in working practices between the two groups.

\section{Which model of care would be most appropriate?}

Three models of care were proposed as improvements on the current system, illustrated in Figs 3-5. There were three variables: ownership of the patient, their location, and the role of education.

Preoperative assessment was also discussed because it forms a significant part of the 'Proactive care of Older People undergoing Surgery' (POPS) model from which evidence supporting geriatrician input into surgical patients was produced. ${ }^{11}$ However, there was little agreement about whether there was anything a geriatrician could contribute here and, therefore, this is not included in the models described below.

The first model of care (Fig 3) describes an expanded role for surgeons, trained to adequately manage older patients themselves without extra clinical input from the geriatrician. The geriatrician's role would be limited to an advisory and educational one only. This was supported only by the surgical trainee.

The second model of care (Fig 4 ) involved the patient remaining on the surgical ward throughout and being managed jointly. This was supported by the majority of participants. There was disagreement about whether the geriatrician should solely provide clinical care or also teach surgical trainees. Some thought it important for geriatricians to be training surgical trainees, regardless of the model of care. Other geriatricians were clear that this should not be part of their remit at all:

I don't think there's a role to teach them. How do you teach somebody how to manage multi-organ failure in a ninety-year-old? You train them in geriatric medicine... so unless they want to be dual-accredited I think it's the role of a physician and in particular, the role of a geriatrician to do that. (Interview 11, orthogeriatrician).

The third model (Fig 5) involved the geriatrician taking over the care of the patient on a medical ward when the immediate surgical problems had been dealt with, with no role for education. Although this model was initially supported by a few participants, on reflection, they all revised this to support the second model. The geriatricians revised their suggestion when they reflected on their own limitations with surgical complications and the benefit of continuity of care from the MDT. The surgeon only supported the joint care model with current resources, although their ideal was different to that of other participants:

In an ideal world, with an ideal job plan, with ideal resources and everything else, I would say do their surgery, put them on 


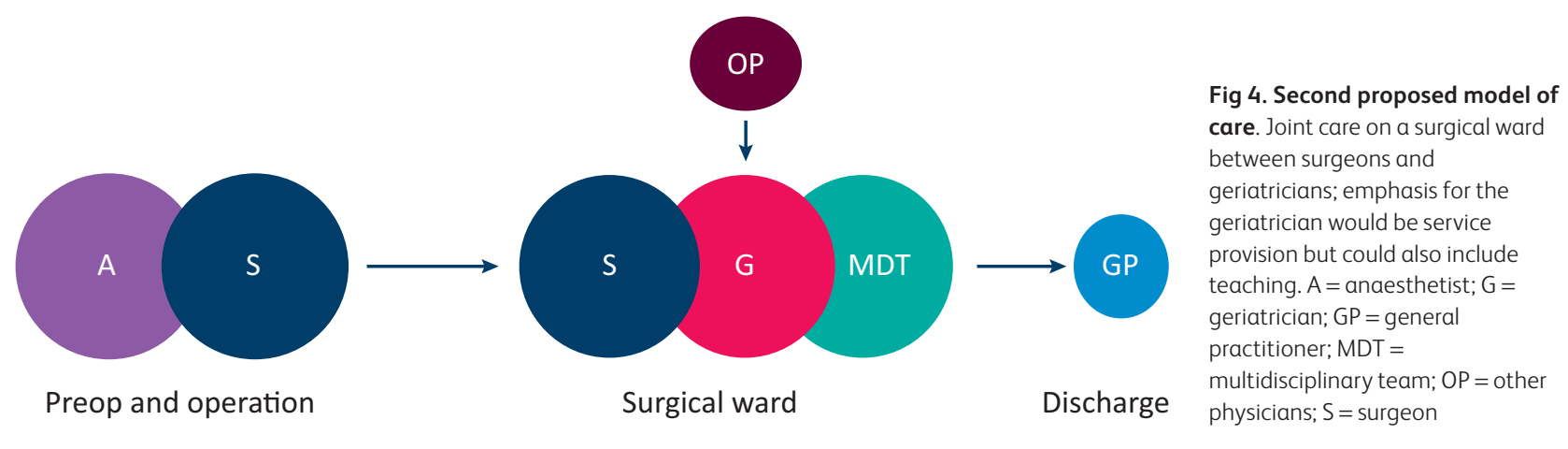

a medical ward and then not our responsibility, it's a medical responsibility (Interview 5, urologist).

\section{Barriers to a change in practice}

\section{Human factors}

Deskilling of surgical doctors. The concern that the introduction of a geriatrician onto a surgical ward would result in deskilling the surgical teams was the most significant barrier identified, even for those surgeons who were broadly supportive of it:

I guess if I had any concerns about geriatricians getting involved it would be regarding the potential further de-skilling of the surgical juniors to do the jobs they should be doing and that we end up being the technicians that just do the operations and then someone else looks after our patients. (Interview 6, general surgeon).

The 'surgeon as technician' was a well-understood concept referred to by four surgeons and, in every case, implied something significantly less than they considered their role to be:

I don't think we were ever tasked to just do the operating and not look after the patients... first of all we're doctors, we all trained. It's easy to...forget that we're actually meant to be doctors first and surgeons in our sub-specialty. (Interview 12, ST6-level surgical trainee).

All those who referred to this term used it to describe the role of the orthopaedic surgeons, often within the orthogeriatric model. Even the orthopaedic surgeon highlighted the difference between his specialty and those of other surgeons:

...orthopaedics is totally different. They don't want to deal with anything medical. General surgery you must appreciate... is the surgical arm of the general physician. And they are good at many things... So there is a bit of an overlap between a general physician and a general surgeon. (Interview 9, orthopaedic surgeon).

The skills that different clinicians value and the overlap with those of the geriatrician was a key theme.

Ontological challenge to surgeons. There were two elements to how the surgeons view themselves that could be challenged by involving a geriatrician in the care of their patients; it appears to question not only their role as the provider of care to their patient, but also their capabilities. Some surgeons talked of 'swallowing their pride' (Interview 6) or having to accept that their outcomes are not as good as they had thought. Two geriatricians could appreciate that it is not easy for a doctor to hear they could be doing better:

If you look at the way that someone gets to being a consultant surgeon, they've been successful by and large with everything they've ever done... therefore, to challenge that and get those people to accept that they need help is difficult. It is... a challenge to what they are. (Interview 7 , orthogeriatrician)

Lack of recognition of need. Most geriatricians reported a lack of understanding of their specialty and patients among other clinicians. First, they felt that some doctors do not recognise what geriatricians do differently to other physicians. As a result, there is little value attached to those skills. Second, the problems encountered with complex older surgical patients can be difficult to recognise. It can be difficult for surgeons to understand the value of a geriatrician if they are not able to identify a problem in the first place.

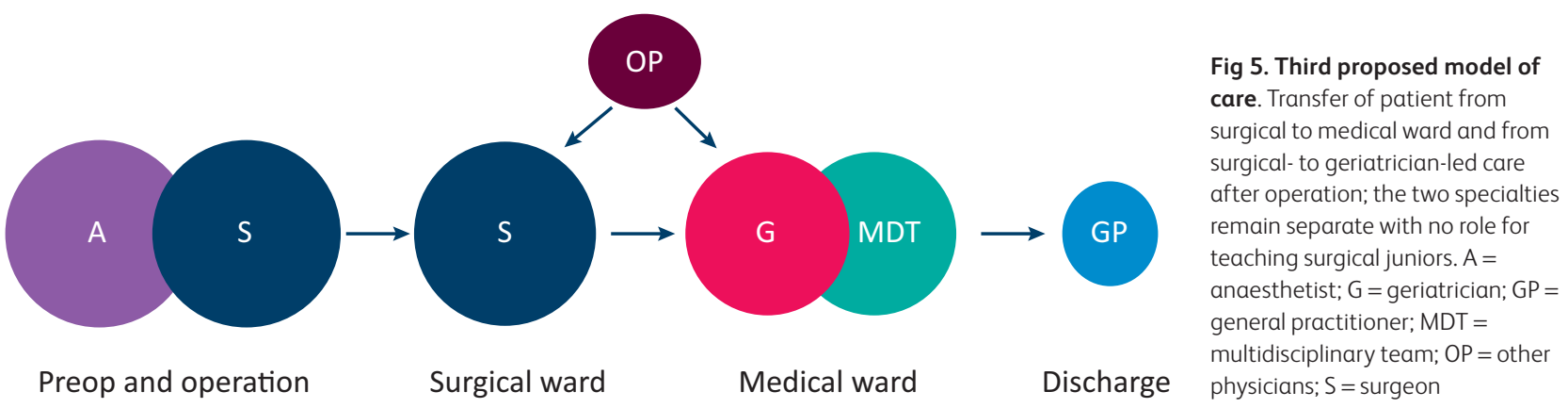


Other barriers

Lack of evidence. Some participants pointed out that the evidence for introducing a geriatrician onto a surgical ward was limited and, without it, there was no current value attached to them. Several geriatricians referred to the POPS data, ${ }^{11}$ with some recognising that it might not translate to smaller hospitals with fewer resources. Other participants from both groups discussed the National Confidential Enquiry into Patient Outcome and Death reports, whereas most referred to the data from the National Hip Fracture Database as evidence that could be applied to other surgical specialties. This is significant given the notable reticence of most of the non-orthopaedic surgeons to consider themselves in the same category as orthopods.

\section{Discussion}

The findings described have examined the problems with the current system and identified a consensus for improvement. The perceived skills of the geriatrician were discussed and the possible models of integration explored. Finally, some of the potential barriers that might hinder closer working practices were identified.

Most felt there was room for improvement in the current system, describing a reactive and fragmented service. Whereas the geriatricians focused on the need to improve the quality of care for this group of patients, the surgeons tended to highlight the delays in discharge that the current system creates. This is relevant for the success of any collaborative service that might rely heavily on length of stay as a key performance indicator.

Although many participants listed some common tangible skills of the geriatrician, two surgeons did not recognise any difference between the geriatrician and any other physician. This suggests a lack of recognition that older patients present particular challenges requiring particular skills. Therefore, it might not be clear to some that a geriatrician is needed at all. It was interesting that the so-called 'surgical culture' was criticised equally by both groups as being task focused and time pressured, with the implication that it compromised patient care. The opposite end of the spectrum appears to be the geriatrician's way of working, which was felt by some to be too broad. A potential challenge to the collaboration of the two groups would be to find a compromise between the two.

Three models of care were put forward to improve on the current system. The key variables were the ownership and location of the patient along with the role of the geriatrician in educating surgical juniors. The first model describes an expanded role for surgeons, trained to manage the changing patient population themselves. This does not sit easily within the surgical culture of restricted and clearly defined boundaries described by many or with suggestions that current surgical training is not sufficient to meet existing expectations. ${ }^{12}$ A survey questioning surgical trainees on their confidence and competence in managing older patients reported that $85 \%$ regularly needing assistance. ${ }^{13}$ This model assumes that it would be possible for a surgical trainee to become competent in both surgery and older patient medicine within a reasonable time period.

The second model, suggested by the majority, involved joint care in some form. This probably reflects recognition that the current boundaries do not facilitate good patient care and that closer collaboration would be preferable. There were differing opinions regarding the role of education here.
The third model involved a change in care and ward postoperatively. Hip fracture data suggest that this model does not confer a benefit and can increase length of stay. ${ }^{14}$ In addition, continuity of care and avoiding moving wards is paramount in key healthcare reports ${ }^{15,16}$ and, as such, it is difficult to envisage that this model could be considered an improvement. However, it is interesting that, in a survey of surgical trainees, $61 \%$ supported this model of care. ${ }^{13}$

The literature suggests that the success of organisational innovations cannot rely on evidence alone and the interests, values and power relationships surrounding the changes should also be considered. ${ }^{17}$ The current study suggests that these human factors are a significant barrier and have not been recognised so far in the literature. The 'surgeon as technician' was a well-understood concept and appeared to be the main concern. Introducing a geriatrician, seen as a generalist rather than a specialist filling a particular gap in care, is seen as someone who will take part of their role away; the geriatrician's remit appears to be too broad for comfort.

Lack of evidence has been highlighted in the literature ${ }^{8}$ and was reiterated in the interviews. Linked to this is the lack of recognition of the need for a geriatrician specifically and is suggested to be related to the lack of understanding of what a geriatrician can offer. Valuing the contribution of holistic care might be difficult in a surgical task-focused, target-driven environment.

\section{Limitations}

The small sample size and limited geographical area do not allow these results to be generalisable beyond this study. The heterogeneity of the surgical specialties is also significant. This study discussed complex older surgical patients in general, but it is clear that different surgical specialties face different challenges with such patients.

\section{Conclusions}

Delivering high-quality care to older surgical patients is a challenge that has to be met. Successful change depends on engaging those involved and the potential barriers discussed in this study are not insignificant. The current literature focuses on the 'evidence' as opposed to the human factors. This study shows that the latter might be just as important in delivering realistic and sustainable change. Those involved in developing services can use this study to inform discussions that are needed to move forward to find the right solution for these complex patients.

\section{References}

1 National Confidential Enquiry into Patient Outcome and Death. Elective and emergency surgery in the elderly: an age old problem. London: NCEPOD, 2010.

2 National Confidential Enquiry into Patient Outcome and Death. Lower limb amputation: working together. London: NCEPOD, 2014.

3 National Confidential Enquiry into Patient Outcome and Death. Extremes of age. London: NCEPOD, 1999.

4 National Confidential Enquiry into Patient Outcome and Death. Knowing the risk. London: NCEPOD, 2011.

5 Patel NK, Sarraf KM, Joseph S et al. Implementing the National Hip Fracture Database: an audit of care. Injury 2013;44:1934-9.

6 Royal College of Physicians. NHFD annual report 2014. London: RCP, 2015.

7 Royal College of Physicians. NHFD 2015 annual report. London: RCP, 2016. 
8 Partridge JSL, Collingridge G, Gordon AL et al. Where are we in perioperative medicine for older surgical patients? A UK survey of geriatric medicine delivered services in surgery. Age Ageing 2014;43:721-4.

9 Healthcare Quality Improvement Partnership. Third Patient Report of the National Emergency Laparotomy Audit (NELA) December 2015-November 2016. London: HQIP, 2017.

10 Hennink M, Hutter I, Bailey A. Qualitative Research Methods. London: Sage, 2011.

11 Harrari D, Hopper A, Dhesi J et al. Proactive care of older people undergoing surgery ('POPS'): designing, embedding, and funding a comprehensive geriatric assessment service for older elective surgical patients. Age Ageing 2007:36:190-6.

12 Thomas C, Griffiths G, Abdelrahman T et al. Does UK surgical training provide enough experience to meet today's training needs? BMJ Careers 2015;350:h2503.

13 Shipway D, Partridge J, Foxton $C$ et al. Do surgical trainees believe they are adequately trained to manage the ageing population? A UK survey of knowledge and beliefs in surgical trainees. J Surg Educ 2015;72:641-7.
14 Parker M, Todd C, Palmar C et al. Inter-hospital variations in length of hospital stay following hip fracture. Age Ageing 1998:27:333-7.

15 Goodwin N, Smith J, Davies A et al. Integrated care for patients and populations: Improving outcomes by working together. London: King's Fund, 2012.

16 Future Hospital Commission. Future hospital: caring for medical patients. London: RCP, 2013.

17 Langley A, Denis J-L. Beyond evidence: the micropolitics of improvement. BMJ Quality Safety 2011;20(Suppl 1):i43-6.

Address for correspondence: Dr Sarah Howie, Kingston Hospital NHS Foundation Trust, Care of the Elderly

Department, Galsworthy Road, Kingston upon Thames KT2 7QB, UK.

Email:s.howie@nhs.net

\section{Royal College} of Physicians

\section{Physicians and war}

War has been a frequent feature of British life over the 500 years of the Royal College of Physicians' existence, and has an important place in the histories of both the RCP and medicine.

Order by phone or online

Tel +44 (0)20 30751358

or visit www.rcplondon.ac.uk/shop

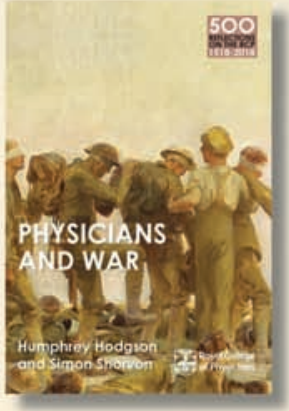

Price: $€ 12$

(Postage and packaging not included)

$10 \%$ discount for fellows and members.

$15 \%$ discount for foundation doctors and students 